\title{
ON THE COMMON RIGHT FACTORS OF MEROMORPHIC FUNCTIONS
}

\author{
Tuen-Wai Ng and Chung-Chun Yang
}

In this paper, common right factors (in the sense of composition) of $p_{1}+p_{2} F$ and $p_{3}+p_{4} F$ are investigated. Here, $F$ is a transcendental meromorphic function and $p_{i}$ 's are non-zero polynomials. Moreover, we also prove that the quotient $\left(p_{1}+p_{2} F\right) /\left(p_{3}+p_{4} F\right)$ is pseudo-prime under some restrictions on $F$ and the $p_{i}$ 's. As an application of our results, we have proved that $R(z) H(z)$ is pseudo-prime for any nonconstant rational function $R(z)$ and finite order periodic entire function $H(z)$.

\section{Introduction and Main Results}

The subject of factorisation under the the composition of meromorphic functions is clearly related to the dynamics of rational or entire functions.

We assume that the reader is familiar with some basic results and notations of Nevanlinna Theory of entire and meromorphic functions (see Hayman [7]), which will be the main tool of our investigations.

In [10], Urabe proved the following theorem.

THEOREM. Let $P_{i}(i=1,2)$ and $Q$ be non-constant polynomials with $\operatorname{deg} P_{1}<$ $\operatorname{deg} P_{2}$ such that $P_{1}(z)-P_{2}(z)$ has only simple zeros. Let

$$
F(z)=\frac{p_{1}(z)+Q\left(e^{z}\right)}{p_{2}(z)+Q\left(e^{z}\right)}
$$

Then $F$ is prime.

Therefore, it is natural to consider the primeness and pseudo-primeness of functions of the general form $\left(p_{1}+p_{2} F\right) /\left(p_{3}+p_{4} F\right)$, where $F$ is a transcendental meromorphic function and the $p_{i}$ 's are polynomials. In this paper, common right factors of functions $p_{1}+p_{2} F$ and $p_{3}+p_{4} F$ (in the sense of composition) will first be investigated. Then we shall also prove that the quotient $\left(p_{1}+p_{2} F\right) /\left(p_{3}+p_{4} F\right)$ is pseudo-prime under some further restrictions on $F$ and the $p_{i}$ 's as follows.

Received 3rd June, 1996

The research was partially supported by a UPGC grant of Hong Kong.

Copyright Clearance Centre, Inc. Serial-fee code: 0004-9729/97 \$A2.00+0.00. 
THEOREM 1. Let $g$ be a common right factor of $p_{1}+p_{2} F$ and $p_{3}+p_{4} F$, where the $p_{i}$ 's are polynomials, $p_{2}, p_{4} \not \equiv 0$ and $F$ is a transcendental meromorphic function. If $\left\{p_{1} p_{4}-p_{2} p_{3}, p_{2}, p_{4}\right\}$ is a linear independent set over $\mathbf{C}$, then $g$ must be a polynomial and is a right factor of $p_{4} / p_{2}$.

THEOREM 2. Let $p_{i}$ be a polynomial for $i=1, \ldots, 4, p_{2}, p_{4} \not \equiv 0$ and let $F$ be a transcendental meromorphic function with at most a finite number of poles. If $p_{2} / p_{4}$ is not a constant, then $\left(p_{1}+p_{2} F\right) /\left(p_{3}+p_{4} F\right)$ is pseudo-prime.

REMARK. If $p_{2} / p_{4}$ is equal to a non-zero constant, then $\left(p_{1}+p_{2} F\right) /\left(p_{3}+p_{4} F\right)$ may not be pseudo-prime. For example, let $f$ be a transcendental meromorphic function such that $F=f\left(e^{z}\right)$ is of finite order. Then $(1+F) /(2+F)=[(1+f) /(2+f)] \circ e^{z}$, which is not pseudo-prime. However, we can still draw the same conclusion under some further restrictions on $F$ and the $p_{i}$ 's.

THEOREM 3. Let $F$ be a periodic entire function of finite order, let $p_{1}, p_{2}$ and $p$ be three polynomials. Let $Q(z)$ be the canonical product of the common roots of $p_{1}+p F$ and $p_{2}+c p F$, where $c$ is a non-zero constant. If $Q / p$ is not a constant, then $\left(p_{1}+p F\right) /\left(p_{2}+c p F\right)$ is pseudo-prime.

COROLlary 1. Let $p_{1}, p_{2}$ be two polynomials, not both constant. Suppose that $H$ is a periodic entire function of finite order. Then $F=p_{1}+p_{2} H$ is pseudo-prime.

PROOF OF COROLlaRY 1: If $p_{2}$ is a constant, it reduces to a result of Gross [4]. Suppose that $p_{2}$ is not a constant. Consider $1+1 / F=\left(p_{1}+1+p_{2} H\right) /\left(p_{1}+p_{2} H\right)$ and apply Theorem 3 to $1+1 / F$ to conclude that $1+1 / F$ is pseudo-prime and hence so is $F$.

As another application of Theorem 3, we can confirm the following result which was conjectured by Zheng in [11].

THEOREM 4. Let $H$ be a periodic entire function of finite order. Then for any non-constant rational function $R(z), F(z)=R(z) H(z)$ is pseudo-prime.

It is natural to conjecture the following :

CONJECTURE. For any non-constant rational function $R(z)$ and periodic entire function $H, R(z) H(z)$ is pseudo-prime.

\section{Some Lemmas}

Lemma 1. [8] Let $f$ be a meromorphic function. Then for all irreducible rational functions in $f$,

$$
R(z, f)=\frac{\sum_{i=0}^{p} a_{i}(z) f^{i}}{\sum_{i=0}^{q} b_{i}(z) f^{i}}
$$


with meromorphic coefficients $a_{p}(z), b_{q}(z) \not \equiv 0$ such that $T\left(r, a_{i}\right)=o(T(r, f))$, $T\left(r, b_{j}\right)=o(T(r, f)) i=0, \ldots, p, j=0, \ldots, q$. Then we have $T(r, R(z, f))=$ $\max (p, q) T(r, f)+o(T(r, f))$.

REMARK. In the sequel, any function $g$ satisfying $T(r, g)=o(T(r, f))$ as $r \rightarrow \infty, r \notin$ $E$ (a set of finite linear measure) will be called a small function of $f$.

LEMMA 2. [1] Let $f$ be a transcendental meromorphic function, $g$ be a transcendental entire function and $R$ be a nonconstant rational function. Then the function $f(g(z))-R(z)$ has infinitely many zeros.

LEMMA 3. [3] Let $f$ be a meromorphic function and $g$ be a transcendental entire function. If $\rho_{f(g)}<\infty$, then $\rho_{f}=0$.

LEMMA 4. [2] Let $f$ be a meromorphic function and $g$ be an entire function such that $\rho_{f(g)}<\infty$. Let $f=f_{1} / f_{2}$, where $f_{1}, f_{2}$ are the canonical products formed by the zeros and poles of $f$ respectively. Then for $\varepsilon>0$ and arbitrary large $r$,

$$
\log M\left(r, f_{1}(g)\right)+\log M\left(r, f_{2}(g)\right)<r^{k}
$$

where $k=(1+\varepsilon)\left(\rho_{f(g)}+\varepsilon\right)+\rho_{g}+\varepsilon$. In particular $\rho_{f_{i}(g)}<\infty$ for $i=1,2$.

In order to investigate the common right factors of the functions $p_{1}+p_{2} F$ and $p_{3}+p_{4} F$, we prove the following lemma.

LEMma 5. Suppose $F$ and $G$ are two transcendental meromorphic functions which satisfy the equation

$$
\sum_{i, j} a_{i j}(z) F^{i}(z) G^{j}(z) \equiv 0
$$

where $a_{i j}(z)$ are linearly independent polynomials over $\mathbf{C}$. If $g$ is a common right factor of $F$ and $G$, then $g$ must be a polynomial.

Proof: Suppose that $g$ is transcendental and write $F=f_{1}(g)$ and $G=$ $f_{2}(g)$, where $f_{1}, f_{2}$ are meromorphic functions. Then the above equation becomes $\sum_{i, j} a_{i j}(z) f_{1}^{i}(g(z)) f_{2}^{j}(g(z)) \equiv 0$. Since $g$ is transcendental and $a_{i j}(z)$ are polynomials, $\sum_{i, j} T\left(r, a_{i j}\right)=o(T(r, g))$. Then we can apply the well known result of Steinmetz ([9] or [5]) to obtain a set of polynomials $P_{i j}(z)$, not all identically zero, such that $\sum_{i, j} a_{i j}(z) P_{i j}(g(z)) \equiv 0$. By rearranging the expression according to the powers of $g$, we have

$$
\sum_{k} c_{k}(z) g^{k}(z) \equiv 0
$$


where $c_{k}(z)$ is a linear combination of the $a_{i j}(z)$ 's over C. By Lemma 1, we must have $c_{k}(z) \equiv 0$ for all the $k$ 's. Since at least one of the $P_{i j}(z)$ is not identically zero, one of the $c_{k}(z)$ is a non-trivial linear combination of the $a_{i j}(z)$ 's over $\mathbf{C}$. This contradicts our hypothesis on the $a_{i j}(z)$ 's.

REMARK. The assumption of linear independence on the $a_{i j}(z)$ 's is essential. For example,

$$
\cos ^{2} z+\sin ^{2} z=1
$$

but $\cos z$ and $\sin z$ have the non-polynomial right common factor $e^{z}$.

The following lemma is implicitly contained in the proof of the result obtained by Gross and Osgood in [6], which is a key factor used in the proof of Theorem 3. By observing that $\sum_{i=1}^{n} T\left(r, h_{i}\right)=o(T(r, g)), \sum_{i=1}^{n} T\left(r, \psi_{i}\right)=o(T(r, g))$, this allows us to choose $M=1$ in [6, p.291]. Together with the lemma in [6, p.288], we can obtain the following result.

LEMmA 6. Suppose that $g$ is a nonconstant entire function, and that $\psi_{i} \not \equiv 0$ for $1 \leqslant i \leqslant n$, are entire functions (where $n \geqslant 2$ is a natural number). Suppose, $f_{i} \not \equiv 0$ and $h_{i} \not \equiv 0(1 \leqslant i \leqslant n)$ are meromorphic functions, with $f_{i}$ analytic at $z=0, f_{1}(0) \neq 0$. Suppose further that $\sum_{i=1}^{n} T\left(r, h_{i}\right)=o(T(r, g))$ and $\sum_{i=1}^{n} T\left(r, \psi_{i}\right)=o(T(r, g))$. If

$$
\sum_{i=1}^{n} f_{i}\left(g \psi_{i}\right) h_{i}(z)=0
$$

then there exist $n$ functions $P_{i}(z, w)$, not all zero, where each $P_{i}(z, w)$ is a polynomial in $w$ whose coefficients are functions of $\psi_{1}, \psi_{2}, \psi_{3}, \ldots, \psi_{n}$, such that

$$
\sum_{i=1}^{n} P_{i}(z, g) h_{i}(z)=0
$$

where $\operatorname{deg} P_{i}(z, w)$ (in $\left.w\right) \leqslant(n-1)$ for $i=1, \ldots, n, P_{1} \not \equiv 0$ and $P_{1} f_{i}\left(\psi_{i} g\right)-P_{i} f_{1}\left(\psi_{1} g\right)$ vanishes to at least the order $n$ at $g=0$.

\section{Proofs of Theorems}

Proof of Theorem 1: Let $p_{1}+p_{2} F=f_{1}(g)$ and $p_{3}+p_{4} F=f_{2}(g)$, where $f_{1}, f_{2}$ are meromorphic functions. Then by eliminating $F$, we have

$$
p_{1}(z) p_{4}(z)-p_{2}(z) p_{3}(z)=p_{4}(z) f_{1}(g(z))-p_{2}(z) f_{2}(g(z)) .
$$

By applying Lemma 5 , one can conclude that $g$ is a non-constant polynomial. Therefore, $f_{1}$ and $f_{2}$ are transcendental since $F$ is transcendental. Since the $p_{i}$ 's are polynomials, 
we still have $\sum_{i=1}^{4} T\left(r, p_{i}\right) \leqslant O(T(r, g))$. Now by the well known result of Steinmetz ([9] or [5]), there exist, polynomials $A(z), B(z)$ and $C(z)$ not all identically zero, such that

$$
\left(p_{1} p_{4}-p_{2} p_{3}\right)(z) C(g(z))=p_{4}(z) A(g(z))-p_{2}(z) B(g(z)) .
$$

By a simple elimination, we conclude from (1) and (2) that

$$
p_{4}(z)\left(f_{1} C-A\right)(g(z))=p_{2}(z)\left(f_{2} C-B\right)(g(z)) .
$$

The fact that $f_{1}, f_{2}$ are transcendental and one of $A, B$ or $C \not \equiv 0$ ensures that $\left(f_{1} C-A\right)(g(z))$ and $\left(f_{2} C-B\right)(g(z))$ are not identically zero. Hence $g$ is a right factor of $p_{4} / p_{2}$.

Proof of Theorem 2: Suppose that $\left(p_{1}+p_{2} F\right) /\left(p_{3}+p_{4} F\right)$ is not pseudoprime. Then $\left(p_{1}+p_{2} F\right) /\left(p_{3}+p_{4} F\right)=f(g)$ where $f$ is a transcendental meromorphic function and $g$ is a transcendental entire function. Therefore,

$$
\begin{aligned}
f(g)-\frac{p_{2}}{p_{4}} & =\frac{p_{1}+p_{2} F}{p_{3}+p_{4} F}-\frac{p_{2}}{p_{4}} \\
& =\frac{p_{1} p_{4}-p_{2} p_{3}}{p_{4}\left(p_{3}+p_{4} F\right)}
\end{aligned}
$$

which has only a finite number of zeros. This contradicts Lemma 2.

Proof of Theorem 3: We may assume $c=1$. Suppose $\left(p_{1}+p F\right) /\left(p_{2}+p F\right)$ is not pseudo-prime. Then $\left(p_{1}+p F\right) /\left(p_{2}+p F\right)=H(K)=H_{1}(K) / H_{2}(K)$, where $H$ is transcendental meromorphic, $K$ is transcendental entire and $H_{1}, H_{2}$ are the canonical products formed by the zeros and poles of $H$ respectively. Note that since $F$ is of finite order, then so is $\left(p_{1}+p F\right) /\left(p_{2}+p F\right)$. Hence $\rho_{H}=\rho_{H_{1}}=\rho_{H_{2}}=0$ by Lemmas 3 and 4. Since $H_{1}$ and $H_{2}$ have no common zeros, neither do $H_{1}(K)$ and $H_{2}(K)$. Therefore, the zeros of $H_{1}(K)$ and $H_{2}(K)$ are zeros and poles of $\left(p_{1}+p F\right) /\left(p_{2}+p F\right)$ respectively. Hence, we have

$$
\begin{aligned}
& p_{1}+p F=H_{1}(K) e^{R} Q \\
& p_{2}+p F=H_{2}(K) e^{R} Q,
\end{aligned}
$$

where $R$ is an entire function and $Q$ is the canonical product of the common roots of $p_{1}+p F$ and $p_{2}+p F$. Clearly, $Q$ is a polynomial and divides $p_{1}-p_{2}$ in the product sense. By Lemma 4 , the order of $H_{1}(K)$ is finite. Therefore from (3), $e^{R}$ is of finite order and hence $R$ must be a polynomial. Subtracting (4) from (3) and then dividing both sides by $e^{R}$, we get

$$
\frac{p_{1}-p_{2}}{Q} e^{-R}=\left(H_{1}-H_{2}\right) \circ K
$$


Clearly $R$ can't be a constant, otherwise $K$ will be a polynomial. Now if $H_{1}-H_{2}$ has more than one distinct zero, then as $K$ is transcendental entire, by Picard's theorem, $\left(H_{1}-H_{2}\right) o K$ would have infinitely many zeros. This is impossible since the number of zeros of $\left(p_{1}-p_{2}\right) / Q$ is finite.

Since the order of $H_{1}-H_{2}$ is zero, we must have $H_{1}-H_{2}=(z-a)^{n}$ and then $K(z)=a+P(z) e^{-R(z) / n}$, where $a$ is a complex number and $P(z)^{n}=\left(p_{1}-p_{2}\right) / Q$ for some natural number $n$. By considering $H_{i}(z+a)$ instead of $H_{i}(z)$, we may assume $a=0$. Since $H_{1}$ and $H_{2}$ have no common zeros, we may also assume $H_{1}(0) \neq 0$. Now

$$
p_{1}(z)+p(z) F(z)=H_{1}\left(P(z) e^{-R(z) / n}\right) e^{R(z)} Q(z) .
$$

Eliminating $F$ by using the fact that $F$ is periodic with period 1 (say), we get

$$
\begin{aligned}
H_{1}\left(P(z+1) e^{-R(z+1) / n}\right) e^{R(z+1)} Q(z+1) p(z) & -H_{1}\left(P(z) e^{-R(z) / n}\right) e^{R(z)} Q(z) p(z+1) \\
& +p_{1}(z) p(z+1)-p_{1}(z+1) p(z) \equiv 0 .
\end{aligned}
$$

If we let $S(z)=R(z+1)-R(z)$ and multiply both sides by $e^{-R(z)}$, then

$$
\begin{aligned}
H_{1}\left(P(z+1) e^{-R(z+1) / n}\right) e^{S(z)} Q(z+1) p(z)-H_{1}\left(P(z) e^{-R(z) / n}\right) Q(z) p(z+1) & \\
& +\left(p_{1}(z) p(z+1)-p_{1}(z+1) p(z)\right) e^{-R(z)} \equiv 0 .
\end{aligned}
$$

Set $f_{1}=H_{1}, \quad f_{2}=H_{1}, \quad f_{3}=z^{n}, \quad h_{1}(z)=e^{S(z)} Q(z+1) p(z), \quad h_{2}(z)=$ $-Q(z) p(z+1)$ and $h_{3}(z)=p_{1}(z) p(z+1)-p_{1}(z+1) p(z)$. Also let $g(z)=e^{-R(z) / n}$, $\psi_{1}(z)=P(z+1) e^{-R(z+1) / n} / e^{-R(z) / n}, \psi_{2}(z)=P(z), \psi_{3}(z)=1$.

Then the above equation becomes

$$
f_{1}\left(\psi_{1} g\right) h_{1}+f_{2}\left(\psi_{2} g\right) h_{2}+f_{3}\left(\psi_{3} g\right) h_{3} \equiv 0 .
$$

Since $\operatorname{deg}(R(z+1)-R(z)) \leqslant \operatorname{deg} R(z), T\left(r, \psi_{1}\right)=o(T(r, g))$ and $T\left(r, h_{1}\right)=$ $o(T(r, g))$. It is then easy to check that $\sum_{i=1}^{3} T\left(r, h_{i}\right)=o(T(r, g))$ and $\sum_{i=1}^{3} T\left(r, \psi_{i}\right)=$ $o(T(r, g))$. Therefore by Lemma 6 , there exist polynomials $P_{1}(z, w), P_{2}(z, w)$ and $P_{3}(z, w)$ of degree $P_{i}$ (in $w$ ) $\leqslant 3-1=2$, with coefficients being functions of $\psi_{i}$, such that,

$$
P_{1}(z, g) h_{1}+P_{2}(z, g) h_{2}+P_{3}(z, g) h_{3} \equiv 0,
$$

where the $P_{i}$ 's satisfy the conditions that

$$
P_{1} f_{i}\left(\psi_{i} g\right)-P_{i} f_{1}\left(\psi_{1} g\right)
$$


vanishes to at least order 3 at $g=0$ for $i=2,3$ and $P_{1} \not \equiv 0$. We rewrite (5) as

$$
P_{1}(z, g) e^{S(z)} Q(z+1) p(z)-P_{2}(z, g) Q(z) p(z+1)+P_{3}(z, g) h_{3} \equiv 0 .
$$

Let $P_{i}(z, g)=\sum_{k=0}^{2} c_{i k}(z) g^{k}$ and $H_{1}(z)=\sum_{j=0}^{\infty} a_{j} z^{j}$. Because $P_{1} f_{2}\left(\psi_{2} g\right)-P_{2} f_{1}\left(\psi_{1} g\right)$ vanishes to at least order 3 at $g=0$, we have for $m=0,1,2$,

$$
\begin{aligned}
& c_{10} a_{m} \psi_{2}^{m}+c_{11} a_{m-1} \psi_{2}^{m-1}+\cdots+c_{1 m} a_{0} \\
& \quad=c_{20} a_{m} \psi_{1}^{m}+c_{21} a_{m-1} \psi_{1}^{m-1}+\cdots+c_{2 m} a_{0}
\end{aligned}
$$

On the other hand, since $P_{1} f_{3}\left(\psi_{3} g\right)-P_{3} f_{1}\left(\psi_{1} g\right) \equiv P_{1} g^{n}-P_{3} f_{1}\left(\psi_{1} g\right)$ which vanishes to at least order 3 at $g=0$, we see that $c_{30} \equiv 0$ as $n \geqslant 1$.

Now rearrange (7) according to the powers of $g$. We get a polynomial equation in $g$ with small functions of $g$ as the coefficients. By Lemma 1, these coefficients must be identically zero and we have for $m=0,1,2$,

$$
c_{1 m}(z) e^{S(z)} Q(z+1) p(z)-c_{2 m}(z) Q(z) p(z+1)+c_{3 m}(z) h_{3} \equiv 0 .
$$

Since $c_{30} \equiv 0$, for $m=0$,

$$
c_{10} e^{S(z)} Q(z+1) p(z)-c_{20} Q(z) p(z+1) \equiv 0 .
$$

From (8), we have $c_{10} a_{0}=c_{20} a_{0}$. Note that $a_{0}=f_{1}(0)=H_{1}(0) \neq 0$. Therefore

$$
c_{10}\left(e^{S(z)} Q(z+1) p(z)-Q(z) p(z+1)\right) \equiv 0 .
$$

If $e^{S(z)} \equiv 1$ and $c_{10} \not \equiv 0$, then $Q(z+1) / p(z+1) \equiv Q(z) / p(z)$ which implies that $Q(z) / p(z)$ is a constant and this contradicts our assumption. Therefore either $e^{S(z)} \not \equiv 1$ or $c_{10} \equiv 0$. If $e^{S(z)} \not \equiv 1$, then $\left(e^{S(z)} Q(z) p(z+1)-Q(z+1) p(z)\right) \not \equiv 0$ and therefore $c_{10} \equiv 0$.

Hence $c_{10}=c_{20}=c_{30}=0$. Because $c_{10}=c_{30}=0$ and the coefficient of $g$ in $P_{1} g^{n}-P_{3} f_{1}\left(\psi_{1} g\right)$ is identically zero we see that $c_{31} \equiv 0$.

From (8) again we have $c_{11} a_{0}=c_{21} a_{0}$. From this together with $c_{31} \equiv 0$, (9) becomes

$$
c_{11}\left(e^{S(z)} Q(z+1) p(z)-Q(z) p(z+1)\right) \equiv 0 .
$$

This allows us to repeat the above argument to conclude that $c_{11}=c_{21}=0$. Then repeating the argument once more, we have $c_{1 i} \equiv 0$ for $i=0,1,2$. This contradicts the fact that $P_{1}(z) \not \equiv 0$. 
Proof of Theorem 4: Let $R(z)=p_{1}(z) / p_{2}(z)$, where $p_{1}$ and $p_{2}$ do not have any common zero. Consider $1+1 / F=\left(p_{2}+p_{1} H\right) /\left(p_{1} H\right)$. Let $Q(z)$ be the canonical product of the common roots of $p_{2}+p_{1} H$ and $p_{1} H$. Then clearly $Q(z)$ divides $p_{2}$ in the product sense. If one of $p_{1}$ or $Q$ is non-constant, then $Q / p_{1}$ is non-constant as $p_{1}$ and $p_{2}$ do not have any common zero. Now by Theorem 3 , we conclude that $1+1 / F$ is pseudo-prime and so is $F$.

If both $p_{1}$ and $Q$ are constants (say $p_{1}=1$ ), then $H$ and $p_{2}+H$ do not have any common zero and therefore $H(z) / p_{2}(z)$ has finitely many poles.

Suppose that $H(z) / p_{2}(z)=f_{1}(g)$, where $f_{1}$ is a transcendental meromorphic function and $g$ is a transcendental entire function. Using the fact that $H(z) / p_{2}(z)$ has finitely many poles, we can conclude that $g(z)=a+P(z) e^{-K(z) / n}$ and $f_{1}(z)=$ $f(z) /(z-a)^{n}$ where $P(z), K(z)$ are polynomials, $K(z)$ is non-constant and $f$ is a transcendental entire function of zero order with $f(a) \neq 0$. By considering $f(z+a)$ instead of $f(z)$, we may assume $a=0$. Note that $P(z)^{n}=p_{2}(z)$ where $n$ is an natural number. Hence, we have $H(z)=f(w) o\left(P(z) e^{-K(z) / n}\right) e^{K(z)}$.

The fact that $H(z)$ is periodic (say with period 1 ) gives

$$
f(\psi(z) g(z)) e^{\beta(z)}-f(g(z))=0
$$

where $\beta(z)=K(z+1)-K(z)$ and $\psi(z)=P(z+1) / P(z) e^{-\beta(z) / n}$ is an meromorphic function with $T(r, \psi)=o(T(r, g))$. By applying Lemma 6 , there exist two polynomials in $w, P_{1}(z, w) \not \equiv 0, P_{2}(z, w)$, with degree $P_{i}$ (in $\left.w\right) \leqslant 2-1=1$ and cofficients being functions of $\psi$, such that

$$
P_{1}(z, g(z)) e^{\beta(z)}-P_{2}(z, g(z))=0 .
$$

Then,

$$
\frac{P_{2}(z, g)}{P_{1}(z, g)}=e^{\beta(z)} .
$$

By Lemma 1, this is impossible if either degree $P_{i}$ (in $w$ ) $\neq 0$. Therefore, both of degree $P_{i}$ (in w) $=0$ and hence $P_{i}(z, g)=c_{i}(z)$ where $c_{i}(z)$ is a function of $\psi$. Using the fact that $P_{1} f(g)-P_{2} f(\psi g)$ vanishes to at least order 2 at $g=0$ and by arguments similar to those in the proof of Theorem 3 , we can conclude that $c_{1} \equiv c_{2}$ and hence $e^{\beta(z)} \equiv 1$. Then Equation (13) becomes

$$
f(\psi(z) g(z))=f(g(z))
$$

where $\psi(z)=P(z+1) / P(z)$.

Now we can choose a constant $A$ such that the equation $\psi(z) g(z)=A$ has infinitely many roots, say $\left\{a_{i}\right\}$. Note that $g\left(a_{i}\right)=A / \psi\left(a_{i}\right)$ which converges to $A$ as $i$ trends to infinity and from (14), $f\left(g\left(a_{i}\right)\right)=f(A)$. By the uniqueness theorem, we have $f(z) \equiv f(A)$ which is impossible. Finally, we conclude that $R(z) H(z)$ is pesudoprime. 


\section{REFERENCES}

[1] W. Bergweiler, 'On the composition of transcendental entire and meromorphic functions', Proc. Amer. Math. Soc. 123 (1995), 215l-2153.

[2] W. Bergweiler and C.C.Yang, 'On the value distribution of composite meromorphic functions', Bull. London Math. Soc. 25 (1993), 357-361.

[3] A. Edrei and W.H.J. Fuchs, 'On the zeros of $f(g(z))$ where $f$ and $g$ are entire functions', J. Analyse Math. 12 (1964), 243-255.

[4] F. Gross, 'On factorization of meromorphic functions', Trans. Amer. Math. Soc. 131 (1968), 215-222.

[5] F. Gross and C.F. Osgood, 'A simpler proof of a theorem of Steinmetz', J. Math. Anal. Appl. 142 (1989), 290-294.

[6] F. Gross and C.F. Osgood, 'An extension of a theorem of Steinmetz', J. Math. Anal. Appl. 159 (1991), 287-292.

[7] W.K. Hayman, Meromorphic functions (Clarendon Press, Oxford, 1964).

[8] A.Z. Mohon'ko, 'The Nevanlinna characteristics of certain meromorphic functions', (in Russian), Teor. Funktsií Funktsional. Anal. i Prilozhen. 14 (1971), 83-87.

[9] N. Steinmetz, 'Über die faktorisierbaren Losungen Gewohnlicher differentialgleichungen', Math. Z. 170 (1980), 169-180.

[10] H.Urabe, 'Some remarks on factorization of meromorphic functions of finite order with a finite number of poles', Bull. Kyoto Univ. Ed. Ser. B 83 (1993), 1-7.

[12] J.H. Zheng, 'On permutability of periodic entire functions', J. Math. Anal. Appl. 140 (1989), 262-269.

Department of Mathematics

Hong King University of Science and Technology

Clear Water Bay

Kowloon

Hong Kong

e-mail: mayang@usthk.ust.hk 